\title{
Cellular Correlates of Long-Term Sensitization in Aplysia
}

\author{
Leonard J. Cleary, Wai L. Lee, and John H. Byrne \\ W. M. Keck Center for the Neurobiology of Learning and Memory, Department of Neurobiology and Anatomy, \\ University of Texas Houston Medical School, Houston, Texas 77225
}

\begin{abstract}
Although in vitro analyses of long-term changes in the sensorimotor connection of Aplysia have been used extensively to understand long-term sensitization, relatively little is known about the ways in which the connection is modified by learning in vivo. Moreover, sites other than the sensory neurons might be modified as well. In this paper, several different biophysical properties of sensory neurons, motor neurons, and LPI17, an identified interneuron, were examined. Membrane properties of sensory neurons, which were expressed as increased excitability and increased spike afterdepolarization, were affected by the training. The biophysical properties of motor neurons also were affected by training, resulting in hyperpolarization of the resting
\end{abstract}

membrane potential and a decrease in spike threshold. These results suggest that motor neurons are potential loci for storage of the memory in sensitization. The strength of the connection between sensory and motor neurons was affected by the training, although the connection between LPI17 and the motor neuron was unaffected. Biophysical properties of LPI17 were unaffected by training. The results emphasize the importance of plasticity at sensory-motor synapses and are consistent with the idea that there are multiple sites of plasticity distributed throughout the nervous system.

Key words: sensitization; facilitation; excitability; plasticity; tail withdrawal; Aplysia
Sensitization is a form of nonassociative learning in which an animal's response to a weak stimulus is enhanced after another strong, usually noxious, stimulus. This form of learning has important advantages for studying the cellular mechanisms underlying behavioral modifications. For example, sensitization affects behaviors that are mediated by well defined neural circuits. Consequently, it is possible to identify major sites of plasticity. Moreover, changes in the properties of circuit neurons are likely to contribute directly to the behavioral modification.

The marine mollusk Aplysia californica has been used extensively as a model system to study cellular mechanisms underlying sensitization. Two defensive withdrawal reflexes have been studied most intensively, the siphon-induced siphon-gill withdrawal reflex and the tail-induced tail-siphon withdrawal reflex (for review, see Cleary et al., 1995). The monosynaptic components of circuits underlying both of these reflexes have been identified, as have several polysynaptic components (Cleary et al., 1995). The duration of sensitization depends on the training protocol (Pinsker et al., 1973; Frost et al., 1985; Scholz and Byrne, 1987). Short periods of training result in behavioral modifications that last for a relatively short time (15-60 $\mathrm{min})$, whereas multiple training sessions occurring over longer periods of time result in more persistent modifications (1-14 d). Although the duration of sensitization appears to be graded, there is a key mechanistic difference between the two forms of sensitization. Only the long-term form is blocked by inhibitors of protein synthesis (Castellucci et al., 1989) (see also Davis and Squire, 1984).

Received Dec. 18, 1997; revised May 15, 1998; accepted May 15, 1998.

This work was supported by National Institutes of Health Grant R01 NS19895 and National Institute of Mental Health Award K05 MH00649 to J.H.B. and by National Science Foundation Grant ISBN 9320549 to L.J.C. We thank Michelle Aguirre, Jason Molpus, Andrew Tang, and Han Zhang for technical assistance with the behavioral training procedures.

Correspondence should be addressed to Dr. John H. Byrne, Department of Neurobiology and Anatomy, University of Texas Houston Medical School, P.O. Box 20708, Houston, TX 77225

Copyright (C) 1998 Society for Neuroscience $\quad 0270-6474 / 98 / 185988-11 \$ 05.00 / 0$
To understand fully the mechanisms underlying learning, it is critical to study changes in the nervous systems of behaving animals, i.e., animals that have been trained in their intact, living state. Ideally, one would use noninvasive techniques to assess neuronal properties of individual animals before and after training. At present, however, a more practical approach is to look for correlates of sensitization by comparing the properties of neurons in trained animals with those in untrained animals. A potential problem with the design of these experiments is the large number of possible changes that can occur in the nervous system and the difficulty of interpreting which among these is necessary for the behavioral modification. By using Aplysia as a model system, we took advantage of the large body of evidence gathered from in vitro preparations to focus our attention on critical elements of the neural circuit mediating the tail-siphon withdrawal reflex and their most important biophysical properties.

In this paper we examined several different biophysical properties of sensory neurons, motor neurons, and LPl17, an identified interneuron, in animals that had been subjected to long-term sensitization training. As observed in other studies, membrane properties of sensory neurons were affected by the training. The biophysical properties of motor neurons also were affected by training, which suggests that these neurons are potential loci for storage of the memory in sensitization. As in previous studies, the strength of the connection between sensory and motor neurons was affected by the training, although the connection between LP117 and the motor neuron was unaffected.

\section{MATERIALS AND METHODS}

\section{Behavioral training}

Aplysia californica weighing 125-500 gm were obtained from Marine Specimens Unlimited (Pacific Palisades, CA) and Alacrity Marine Biological (Redondo Beach, CA). Animals were parapodectomized to permit full visualization of the siphon. The strength of the tail-induced siphon withdrawal reflex was tested by delivery of a weak electrical stimulus through Teflon-coated silver electrodes (type Ag 5T, Medwire) implanted under the skin in the posterior region of the tail, as described 
A

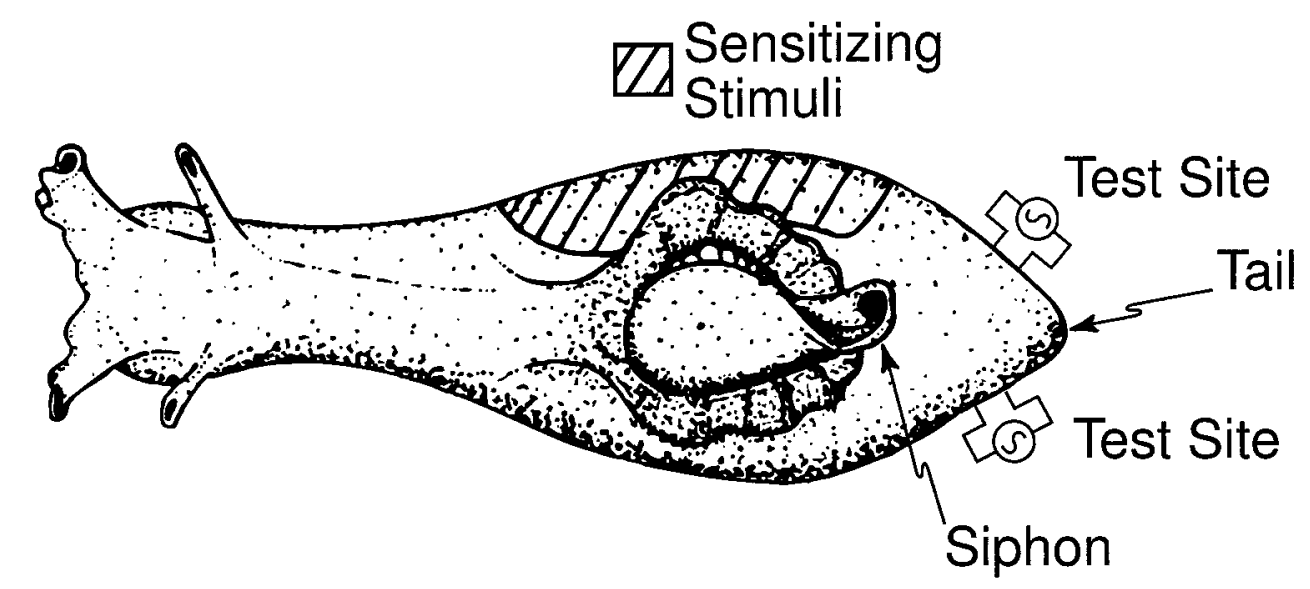

B Testing and Training Protocol

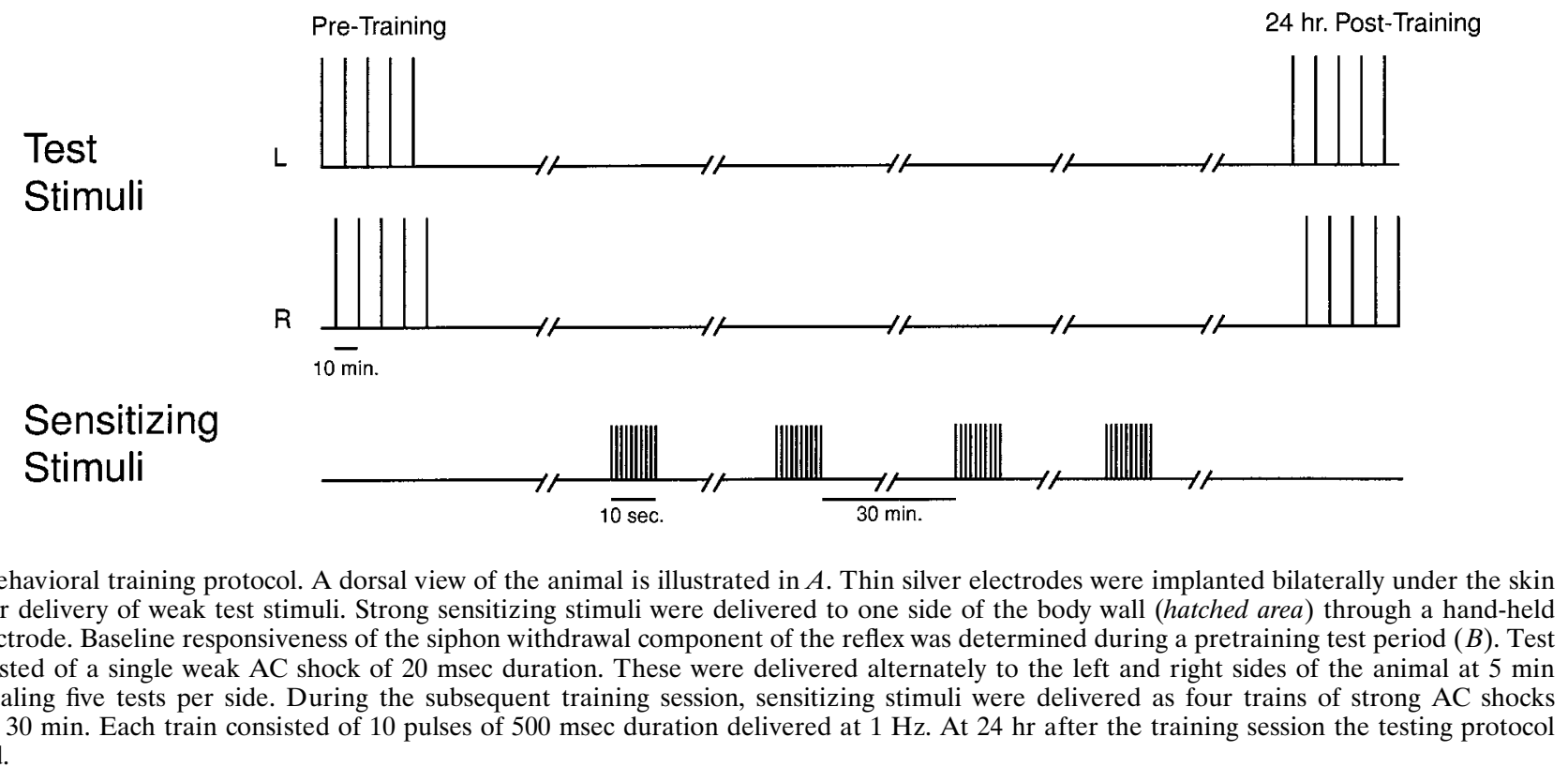

previously (Fig. 1) (Scholz and Byrne, 1987; Goldsmith and Byrne, 1993). Each test stimulus consisted of a $20 \mathrm{msec}$ AC shock provided by a Variac for which the output was gated by a relay. The output of the Variac passed through a resistance of $20.9 \mathrm{k} \Omega$ to provide a quasi constant current. The maximum short-circuit current that could be passed through this circuit for testing stimuli was $\sim 6 \mathrm{~mA}$. The stimulus voltage was set at two times the threshold intensity level necessary to elicit a siphon withdrawal. Ten tests were delivered at intervals of $5 \mathrm{~min}$ to alternating sides (i.e., five tests were delivered per side). The duration of the siphon withdrawal was measured from the start of siphon contraction until the start of relaxation. Relaxation was defined as the reextension of the siphon to its original position. These weak tail stimuli also elicited a withdrawal of the tail, but this response was difficult to quantify and was not measured in these studies. Several criteria were in place at this stage of the experiment to detect and exclude unsuitable animals: inking while acclimating to the test bowl $(n=4)$, inking in response to test stimuli $(n=0)$, and threshold exceeding $3 \mathrm{~mA}(n=0)$.

For analysis the five responses were combined to provide an average withdrawal duration. For tests before training the 5 min interval was sufficient to prevent frequency-dependent changes in the response during the pretraining session. After training, however, the responses at the end of the posttraining session were somewhat greater than those earlier in the session. This probably did not contribute to lateralized enhance- ments induced by training, because both sides exhibited the frequencydependent enhancement.

Sensitizing stimuli were applied to the region of the body wall lateral to the parapodium (Fig. 1). This region was anterior to the area in which the test electrodes were implanted and excluded receptive fields of tail sensory neurons (Walters et al., 1983; Dulin et al., 1995). A hand-held spanning electrode (1 mm gap, $1 \mathrm{~mm}$ electrode length) was used to deliver four trains of strong AC shocks. Each train consisted of 10 shocks of $500 \mathrm{msec}$ duration at $1 \mathrm{~Hz}$. The output of the Variac was passed to the spanning electrode through a resistance of $940 \Omega$ and was set to deliver a short-circuit current of $60 \mathrm{~mA}$. Shocks of this strength reliably produced an inking response. The amount of ink that was released tended to decrease during the training session as the store was depleted. A single strong shock of $500 \mathrm{msec}$ duration did not produce any visible sign of damage to the skin either immediately after training or $24 \mathrm{hr}$ later. To minimize any possible cumulative damage over the 10 successive shocks, we systematically moved the electrode to 10 different locations on the lateral surface of the body wall. Each training session consisted of four trains of 10 shocks delivered at intervals of $30 \mathrm{~min}$. Only one side of the animal received training; the contralateral side served as control. The side to be trained was determined by coin flip at the start of the session.

At a period $24 \mathrm{hr}$ later the strength of siphon withdrawal was tested again, using the protocol described above. The strengths of the testing 


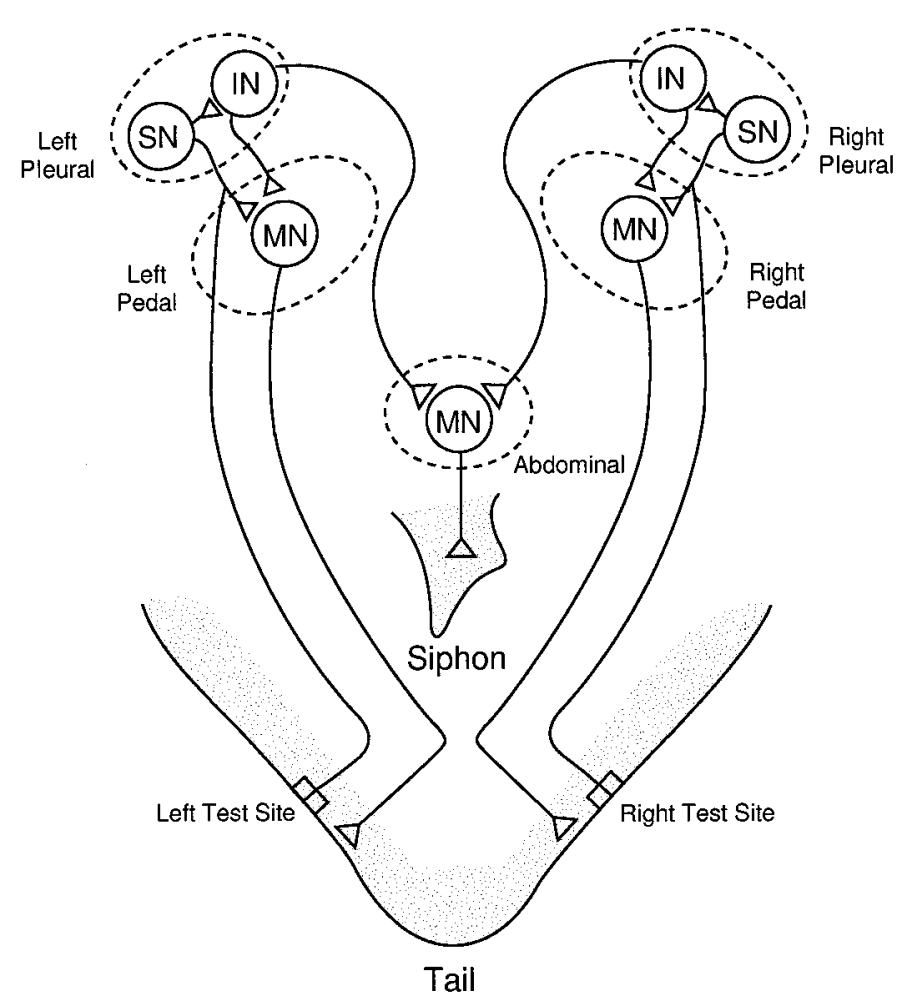

Figure 2. Schematic representation of the neural circuits underlying the tail-elicited tail-siphon withdrawal reflex. Stimulation of the tail of the animal activates sensory neurons $(S N)$ located in the pleural ganglion. These neurons activate motor neurons $(M N)$ in the pedal ganglion that produce tail withdrawal. In addition, sensory neurons activate a polysynaptic pathway that projects to the abdominal ganglion, resulting in siphon withdrawal. The interneuron (IN) LPl17 is an element of this pathway.

stimuli were exactly the same as those used the previous day. The individual who performed the siphon withdrawal tests did not know which side of the animal had been sensitized.

\section{Intracellular recordings}

Immediately after the post-training test the animals were anesthetized with isotonic $\mathrm{MgCl}_{2}$, and the left pleural pedal ganglia were removed. Therefore, animals in the sensitized group were trained on the left side of the animal, and animals in the control group were sensitized on the right side. Then the ganglia were treated with $0.5 \%$ glutaraldehyde in artificial seawater for $40 \mathrm{sec}$ to immobilize the connective tissue (Mirolli and Gorman, 1968; Byrne et al., 1979). The sheath overlying the pleural pedal ganglia was removed surgically in a solution containing 50\% isotonic $\mathrm{MgCl}_{2}$ and $50 \%$ artificial seawater. Intracellular recordings were made through glass microelectrodes with a resistance of 3-10 $\mathrm{M} \Omega$. Sensory and motor neurons innervating the tail were identified by their size and position in the pleural pedal ganglia (Walters et al., 1983). Sensory neurons used in this study were localized in the medial portion of the ventrocaudal cluster of the pleural ganglion, where sensory neurons innervating the tail are concentrated (Fig. 2) (Walters et al., 1983; Zhang et al., 1993). Neurons in this region also have a high probability of forming monosynaptic connections with tail motor neurons in the pedal ganglion. Sensory neurons were impaled with one electrode; motor neurons were impaled with two to control membrane potential. LPl17 was identified by its position in the pleural ganglion and its ability to evoke a characteristic slow EPSP in the tail motor neuron (Cleary and Byrne, 1993). The individual performing the intracellular recordings did not know which side of the animal had been sensitized nor the results of the testing procedure.

\section{Testing protocol}

The cellular properties of sensory, motor, and interneurons were tested by a standardized protocol.

Sensory neurons and motor neurons. First, a sensory neuron and a follower motor neuron were impaled. The resting membrane potentials of both cells were recorded after a brief period of stabilization (2-5 min). Then we held the membrane potential of the motor neuron manually at $-80 \mathrm{mV}$ by passing current through the second electrode. Depolarizing current pulses of gradually increasing intensity $(0.1 \mathrm{nA}$ steps, $50 \mathrm{msec}$ duration) were applied to the sensory neuron until the cell fired, evoking an EPSP. The number of spikes elicited by the short current pulse was recorded, as was the amplitude of the afterdepolarization. The EPSP was evoked two additional times at intervals of $5 \mathrm{~min}$, and the amplitudes were averaged. This procedure minimized the contribution of other activity-dependent phenomenon such as posttetanic potentiation (PTP) or long-term potentiation, because the pulse elicited more than one spike in only two cases. Sensory neuron excitability was tested by passing a long depolarizing current pulse $(2 \mathrm{nA}, 1 \mathrm{sec})$ and recording the number of evoked action potentials. Input resistance of both sensory and motor neurons was the next parameter to be measured. This parameter was measured by evoking voltage transients with a series of hyperpolarizing current pulses. The amplitude of the hyperpolarizing steps was chosen on a cell-by-cell basis and depended on the input resistance. Generally, cells were tested in increments of $0.1-0.3 \mathrm{nA}$, resulting in voltage steps from approximately -2 to $-40 \mathrm{mV}$. The input resistance was calculated from the slope of the linear portion of the resulting $I-V$ curve.

Interneurons and motor neurons. After the sensory-motor series a second series of measurements was made for interneurons and motor neurons. The holding potential of the motor neuron was adjusted to -80 $\mathrm{mV}$, and excitability was tested by measuring the threshold for evoking an action potential. The amplitude of a depolarizing current pulse was increased in steps $(0.1 \mathrm{nA}, 50 \mathrm{msec})$ until an action potential was evoked. The threshold was recorded as the maximum level of depolarization that did not evoke an action potential. After these tests the excitatory interneuron LP117 was identified by its production of a slow EPSP in the motor neuron. After a $15 \mathrm{~min}$ rest period the input resistance of the interneuron was tested with a series of hyperpolarizing pulses as described above. Five minutes later the amplitude of the slow EPSP evoked in the motor neuron by the interneuron was measured. The interneuron was stimulated with a series of depolarizing current pulses of increasing amplitude $(1,2,4,6,8,10,12,15,18$, and $21 \mathrm{nA})$. Because some interneurons evoked a fast component of the EPSP, which could mask the peak of the slow component, the value of the EPSP at $1 \mathrm{sec}$ after the stimulus was used for analysis. The magnitude of the EPSP was quantified by the slope of the linear portion of the resulting stimulus-response curve.

\section{Statistical analysis}

Data were represented by the median and interquartile range (IR). The Wilcoxon test (behavior) and the Mann-Whitney test (cellular correlates) were calculated by the Prism statistical package (GraphPad Software, San Diego, CA). More animals were tested behaviorally than were tested physiologically, because the cellular experiments could not be completed for all ganglia. In some cases this was attributable to the inability to find a monosynaptic connection. In others it was attributable to recorded cells not surviving the testing protocol. Similarly, more preparations were used for analysis of the sensory-motor connection than the LP117-motor neuron connection, primarily because the success rate for LP117 identification was lower.

\section{RESULTS}

\section{Long-term sensitization training produced a lateralized enhancement of the tail-induced siphon withdrawal reflex}

Before training, the median duration of siphon withdrawal was $\sim 3.5 \mathrm{sec}$ for both sides of the animal (control: $3.44 \mathrm{sec}, 3.0 \mathrm{IR}$; sensitized: $3.55 \mathrm{sec}, 2.5 \mathrm{IR}$ ). The difference was not significant $\left(T_{42}=340 ; p=0.16\right)$.

The response of the siphon to weak tail shock was tested $24 \mathrm{hr}$ after sensitization training. The response elicited by test stimuli on the side of the animal ipsilateral to the training stimulus was markedly enhanced as compared with the pretraining response (Fig. 3). The average ratio of post-test response to pretest response was $186 \%$ (95 IR) for the sensitized side. Conversely, the response elicited by test stimuli contralateral to the trained side 


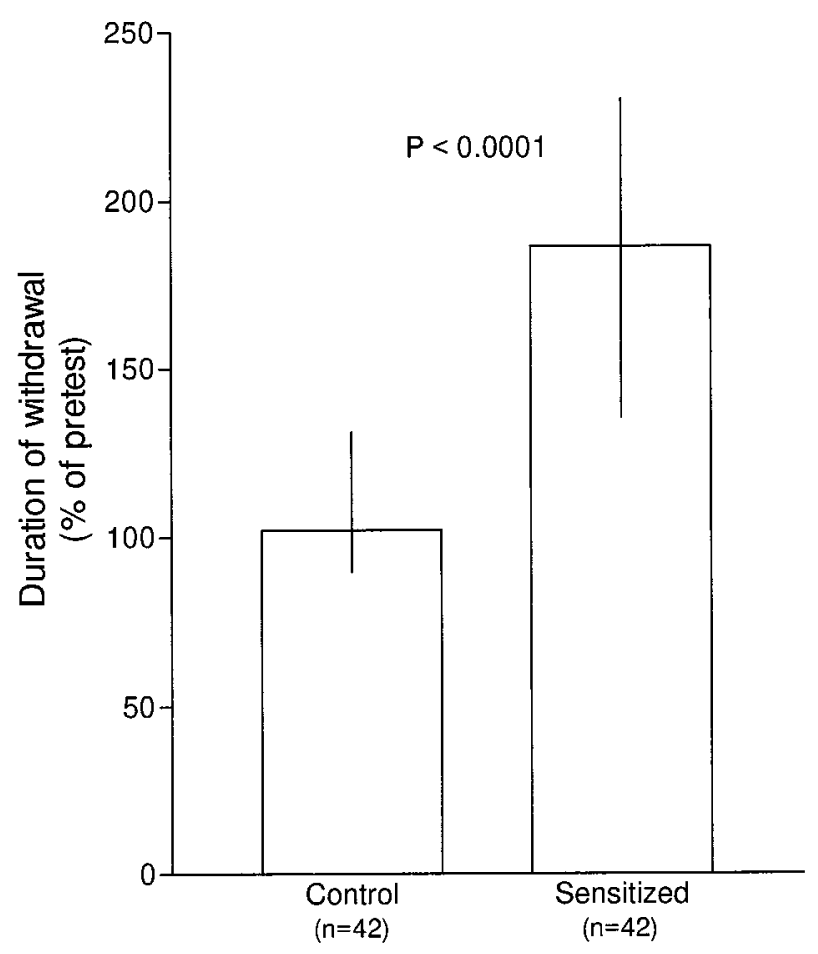

Figure 3. Long-term sensitization training increased the duration of the tail-induced siphon withdrawal reflex. The duration of siphon withdrawal observed during the post-training tests was expressed as a percentage of the pretest duration. Learning was assessed by comparing the normalized duration on the trained side of the animal (Sensitized) with that on the contralateral untrained side (Control).

was not affected. The average ratio of post-test response to pretest response was $102 \%$ (41 IR). The enhancement on the sensitized side of the animal was significantly greater than that on the control side $\left(T_{42}=61 ; p<0.0001\right)$. These results demonstrate that the protocol used in this study produced long-term sensitization of the tail-induced siphon withdrawal reflex. Moreover, the enhancement was lateralized; that is, it was observed only when test stimuli were applied to the trained side of the animal. From these results we infer that the tail withdrawal component of the reflex was enhanced as well, although it was not measured directly.

A critical step in elucidating the cellular mechanisms that mediate this form of learning is to identify the sites of plasticity within the nervous system. Key elements of the circuit mediating the reflex have been identified (see Fig. 2). The pleural sensory neurons make monosynaptic contacts to motor neurons in the pedal ganglion. They also form polysynaptic pathways to motor neurons in the abdominal ganglion that mediate the siphon withdrawal component of the response. The interneurons that project to the abdominal ganglion have not been characterized fully. An excitatory interneuron LP117 has been shown to excite interneurons and motor neurons in the abdominal ganglion contributing to siphon withdrawal (Cleary and Byrne, 1993). This neuron also forms a parallel circuit from tail sensory neurons to tail motor neurons. The inhibitory interneuron RPl4 also forms a parallel circuit to tail motor neurons (Xu et al., 1994), but its effects on siphon motor neurons are unknown.

Modulation of the intrinsic cellular properties of any of these cells could contribute to modulation of the reflex by altering their firing properties. Perhaps more important is the possibility that modulation of synaptic strength anywhere in the circuit, including sensory-motor, sensory-interneuron, and interneuron-motor, could have a large effect on motor neuron output (White et al., 1993). Therefore, we examined the properties of identified neurons in each of these classes and the connections between them.

\section{Long-term sensitization training affects excitability of the tail sensory neurons}

Previous studies demonstrated that long-term sensitization training affected biophysical properties of tail sensory neurons (Scholz and Byrne, 1987). Specifically, training reduced the net outward current elicited by a depolarizing voltage step, an observation consistent with modulation of the S-potassium current. As in short-term sensitization, modulation of the S-current would be expected to increase the excitability of tail sensory neurons (Klein et al., 1986; Baxter and Byrne, 1989). This expectation was confirmed in the present study.

Sensory neuron excitability was measured as the number of action potentials elicited by a depolarizing current pulse of constant amplitude and duration ( $2 \mathrm{nA}, 1 \mathrm{sec})$. The number of action potentials elicited per pulse increased from a median of seven spikes (7 IR) in ganglia from the control side of the animal to 14 spikes (8.5 IR) in ganglia from the sensitized side (Fig. 4A). This difference was statistically significant $\left(U_{19,19}=267 ; p<0.01\right)$. The increase in excitability was expressed primarily as antiadaptation. Sensory neurons in ganglia from the control side stopped firing early in the pulse, whereas sensory neurons in ganglia from the sensitized side fired throughout the pulse.

Another biophysical property of sensory neurons contributing to excitability is the afterdepolarization. The amplitude of the afterdepolarization that follows both the long pulse described above and a short $(50 \mathrm{msec})$ pulse was examined also. In both of these circumstances the amplitude of the afterdepolarization was increased in ganglia from the sensitized side. After the long pulse (1 sec) the afterdepolarization increased from a median of 0.8 $\mathrm{mV}$ (1.8 IR) in ganglia from the control side to $2.6 \mathrm{mV}$ (4.2 IR) in ganglia from the sensitized side $\left(U_{19,19}=260 ; p<0.02\right)$ (Fig. $4 B)$. The afterdepolarization that follows a single spike was affected, as well (Fig. 4C). The afterdepolarization increased from a median of $1.3 \mathrm{mV}$ (1.7 IR) in ganglia from the control side to $2.5 \mathrm{mV}$ (3.8 IR) in ganglia from the sensitized side $\left(U_{18,21}=\right.$ 260; $p<0.01)$.

Long-term sensitization training had no significant effect on the resting membrane potential or input resistance of the tail sensory neurons (Table 1).

\section{Long-term sensitization training affects two biophysical properties of tail motor neurons}

Little is known about the effects of long-term sensitization training on follower motor neurons. Therefore, we examined several biophysical properties of the tail motor neuron. Resting membrane potentials were recorded 5 min after impalement of the neuron with the microelectrode. On average, motor neurons were hyperpolarized after long-term sensitization training (Fig. 5A). The resting membrane potential decreased from a median of -59 $\mathrm{mV}$ (9 IR) in ganglia from the control side to a median of -63 $\mathrm{mV}$ (6 IR) in ganglia from the sensitized side $\left(U_{19,21}=254\right.$; $p<0.04)$.

The effects on membrane potential are counterintuitive, because hyperpolarization would tend to make the motor neuron more difficult to fire under circumstances when it should be firing more intensely. The hyperpolarization may be balanced in part by 


\section{A1. Excitability}

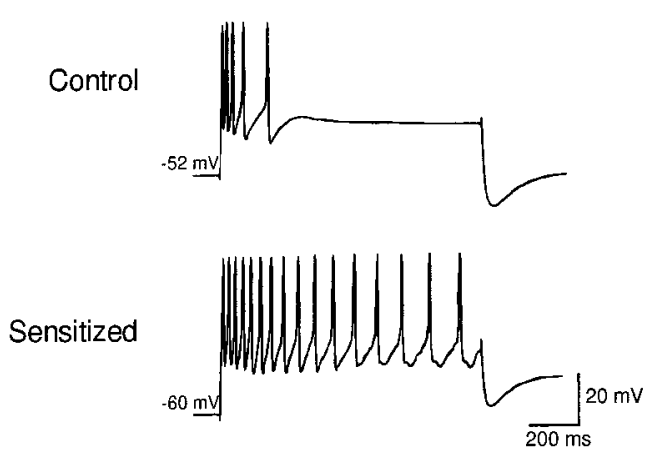

A2

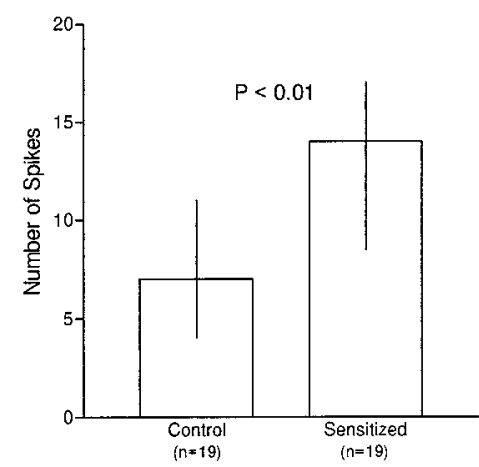

B1. Afterdepolarization (1 s pulse)
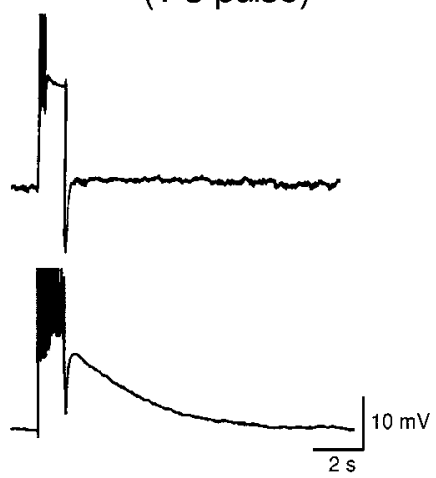

B2

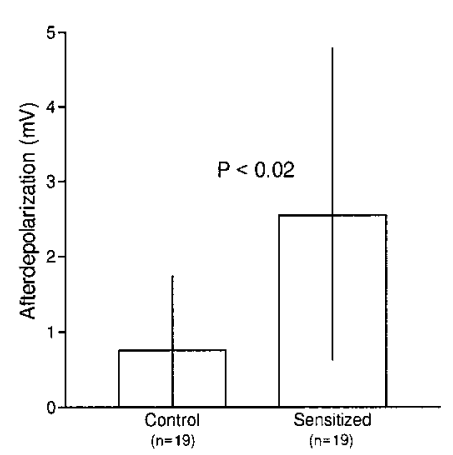

\section{C1. Afterdepolarization (50 ms pulse)}
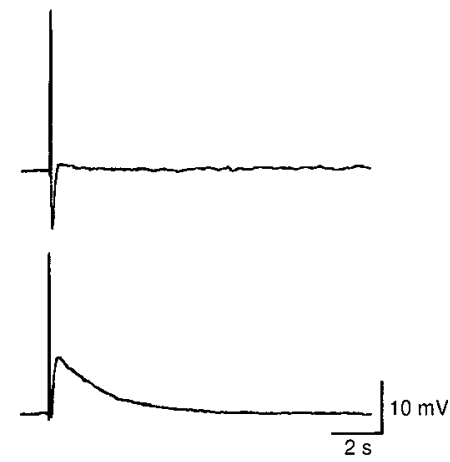

C2

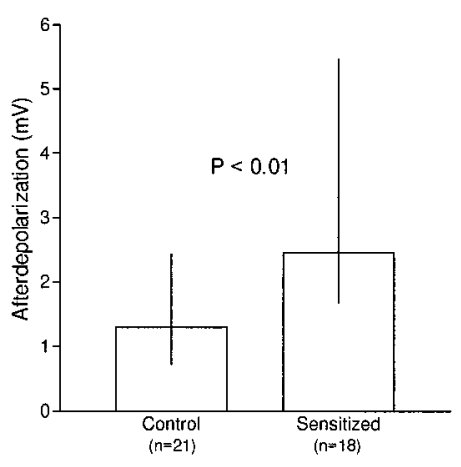

Figure 4. Long-term sensitization produced changes in three biophysical properties of tail sensory neurons: excitability $(A)$, the amplitude of the afterdepolarization that follows a $1 \mathrm{sec}, 2 \mathrm{nA}$ depolarization $(B)$, and the amplitude of the afterdepolarization that follows a $50 \mathrm{msec}$, $0.5 \mathrm{nA}$ depolarization (C). In Panels 1 (top), recordings from both control and sensitized animals are shown. In Panels 2 (bottom), the group data are shown. In $B$ and $C$, the examples were chosen to emphasize the differences.

\begin{tabular}{|c|c|c|c|}
\hline Correlate & Control & Sensitized & \\
\hline $\begin{array}{l}\text { Resting membrane } \\
\text { potential }\end{array}$ & $\begin{array}{l}-50.8(5.5 \mathrm{IR}) \\
(n=22)\end{array}$ & $\begin{array}{l}-54.0 \mathrm{mV}(7 \mathrm{IR}) \\
(n=20)\end{array}$ & $U_{20,22}=253$ \\
\hline Input resistance & $\begin{array}{l}50.2 \mathrm{M} \Omega(29 \mathrm{IR}) \\
(n=19)\end{array}$ & $\begin{array}{l}48.4 \mathrm{M} \Omega(32.6 \mathrm{IR}) \\
(n=20)\end{array}$ & $U_{19,20}=207$ \\
\hline
\end{tabular}

a decrease in the threshold for spike generation. The threshold was determined by gradually increasing the strength of a depolarizing current pulse in $0.1 \mathrm{nA}$ steps at intervals of $1 \mathrm{sec}$ from a fixed holding potential of $-80 \mathrm{mV}$. The threshold was defined as the membrane potential reached by the largest current pulse that did not elicit a spike. Long-term sensitization decreased the threshold for spike initiation (Fig. 5B). The average threshold decreased from a median of $-44 \mathrm{mV}$ (9 IR) to a median of -46 $\mathrm{mV}(6 \mathrm{IR})\left(U_{15,16}=171 ; p<0.05\right)$.

The decrease in spike threshold suggested that membrane excitability of the motor neuron was enhanced. This possibility was tested more directly in some motor neurons by delivering depolarizing current pulses of $3 \mathrm{sec}$ duration in a series of increasing amplitudes of $0.5 \mathrm{nA}$ steps at intervals of 30-70 sec.
Then the number of resulting spikes was counted. Excitability was defined as the slope of the linear portion of the input-output curve. Although there was a strong trend toward increased motor neuron excitability, the enhancement was not statistically significant (Table 2). Moreover, there was a trend toward decreased input resistance of the tail motor neuron after long-term sensitization training, but this modulation was also not statistically significant (Table 2).

\section{Long-term sensitization training increased the strength of the sensorimotor synapse}

The efficacy of sensory neuron transmission was assessed by measuring EPSPs evoked in tail motor neurons. EPSPs were measured with the membrane potential of the motor neuron held at $-80 \mathrm{mV}$ to prevent the motor neuron from firing and to control for possible effects of long-term sensitization on the resting potential of motor neurons (Fig. 6A). After long-term sensitization training, the EPSP increased from a median of $6.9 \mathrm{mV}$ (7.3 IR) in ganglia from the control side to a median of $11 \mathrm{mV}$ (7.2 IR) in ganglia from the sensitized side, but this difference was not statistically significant $\left(U_{17,20}=212 ; p=0.21\right)$. To reduce the variability, we calculated synaptic currents by dividing the amplitude of the EPSP by the input resistance of the motor neuron. The EPSC increased from a median of $0.53 \mathrm{nA}(0.86 \mathrm{IR})$ in 
A1. Resting Membrane Potential

Control

Sensitized

$-59 \mathrm{mV}$

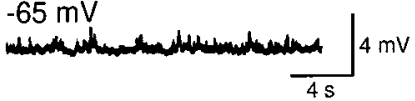

A2

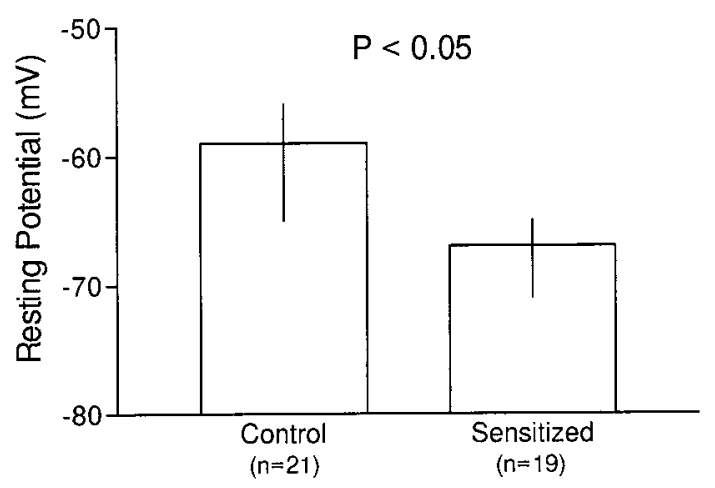

B1. Spike Threshold

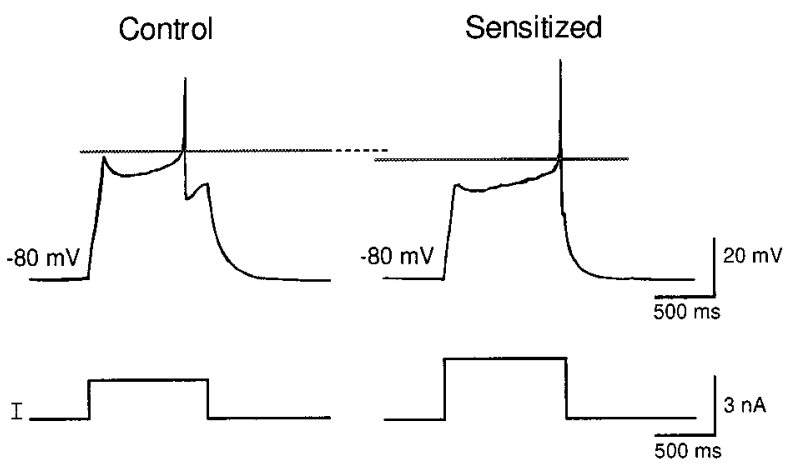

B2

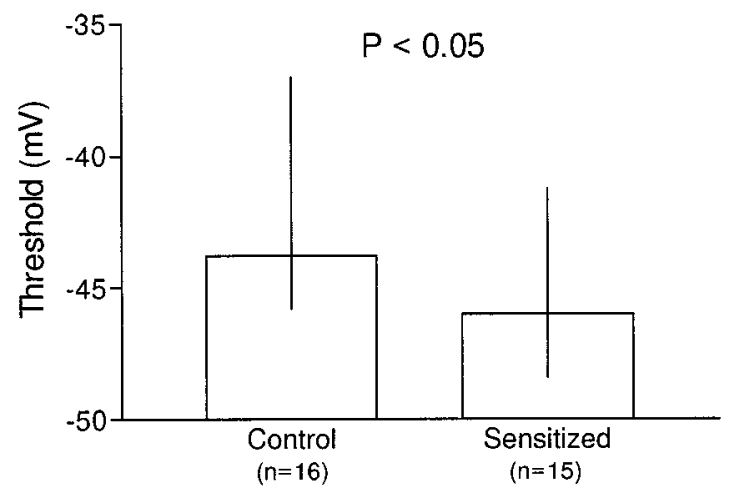

Figure 5. Long-term sensitization produced changes in two biophysical properties of tail motor neurons: resting membrane potential $(A)$ and threshold for spike initiation $(B)$. In Panels 1 (top), recordings from both control and sensitized animals are shown. In Panels 2 (bottom), the group data are shown. In B1, the threshold for each neuron is illustrated by a solid line. The solid line in the Control trace was extended by a dashed line to facilitate comparison. Note that threshold was defined as the largest depolarization that did not initiate a spike. Therefore, threshold was not determined from these traces.

\begin{tabular}{|c|c|c|c|}
\hline Correlate & Control & Sensitized & \\
\hline Input resistance at $-80 \mathrm{mV}$ & $\begin{array}{l}12.7 \mathrm{M} \Omega(6.2 \mathrm{IR}) \\
(n=18)\end{array}$ & $\begin{array}{l}9.1 \mathrm{M} \Omega(2.7 \mathrm{IR}) \\
(n=17)\end{array}$ & $U_{17,18}=195$ \\
\hline Excitability & $\begin{array}{l}4.4 \text { spikes/nA (2.5 IR }) \\
(n=8)\end{array}$ & $\begin{array}{l}7.6 \text { spikes/nA (4.7 IR) } \\
(n=11)\end{array}$ & $U_{8,11}=63$ \\
\hline
\end{tabular}

ganglia from the control side to a median of $1.01 \mathrm{nA}(1.06 \mathrm{IR})$ in ganglia from the sensitized side $\left(U_{16,18}=202 ; p<0.05\right)$ (Fig. $\left.6 B\right)$.

In a previous study the percentage of sensory neurons making connections to motor neurons appeared to increase as a result of long-term sensitization (Frost et al., 1985). The input of tail sensory neurons onto a specific follower is not amenable to this type of analysis, however. There are more neurons in the pleural sensory cluster than in the abdominal LE cluster, and only a small percentage converges onto an individual tail motor neuron.

In ganglia from the sensitized side there was a correlation between the amplitude of the EPSC and the duration of siphon withdrawal ( $r=0.59$, after $\log$ transformation of the data) (Fig. 7). This correlation did not hold for ganglia from the control side. Therefore, the strength of the behavioral response on the control side of the animal appeared to depend on many factors. The enhanced response on the trained side appeared to depend, at least in part, on enhanced transmission from tail sensory neurons.
Synaptic connections from sensory neurons to interneurons probably were modulated to the same extent. We attempted to test this hypothesis directly by examining the amplitude of the EPSP evoked in the interneuron LPl17 by the sensory neuron. The success rate was too low for analysis, however.

\section{Long-term sensitization training did not affect the excitatory interneuron LPI17}

LP117 is an identified interneuron that has widespread effects throughout the CNS (Cleary and Byrne, 1993). Perhaps most importantly, it forms another pathway parallel to the monosynaptic component of the tail withdrawal circuit. Therefore, modulation of LP117 output could have a significant impact on motor neuron activation (White et al., 1993). Also, it activates siphon motor neurons in the abdominal ganglion. Therefore, it could be an additional site for enhancement of siphon withdrawal beyond sensory neuron facilitation and enhanced excitability. Three bio- 
A

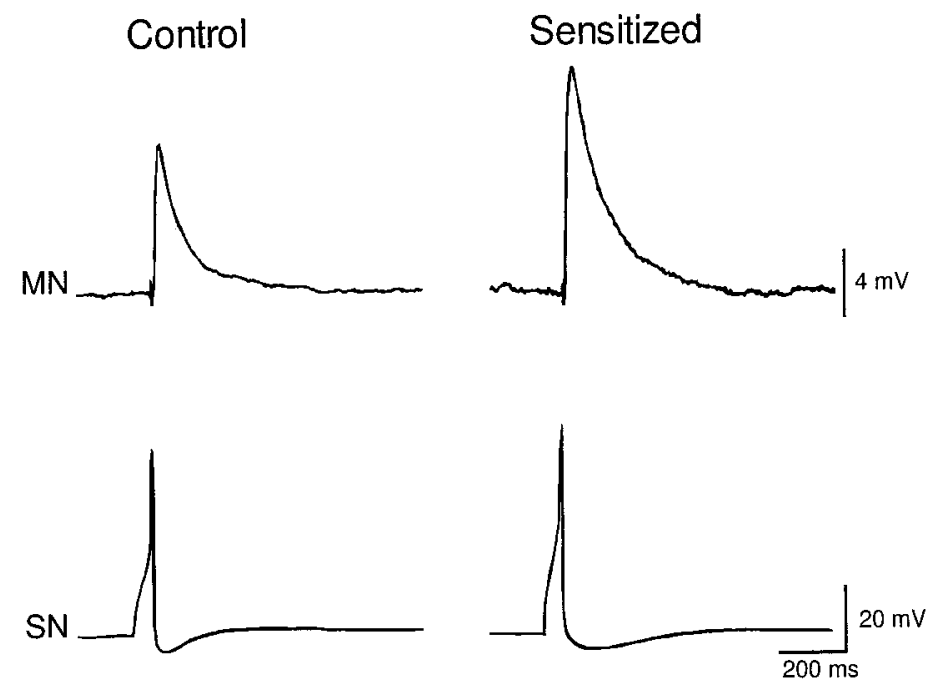

B

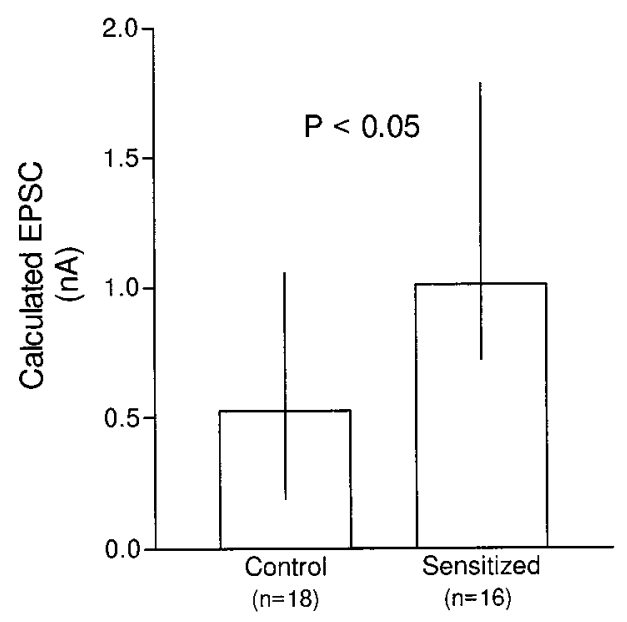

Figure 6. Long-term sensitization training increased synaptic strength between sensory and motor neurons. Recordings of EPSPs evoked in motor neurons by sensory neurons are shown in $A$. In this experiment the motor neurons were held at $-80 \mathrm{mV}$. For the purposes of comparing group data, the EPSC was calculated by normalizing the EPSP by the input resistance of the motor neuron.

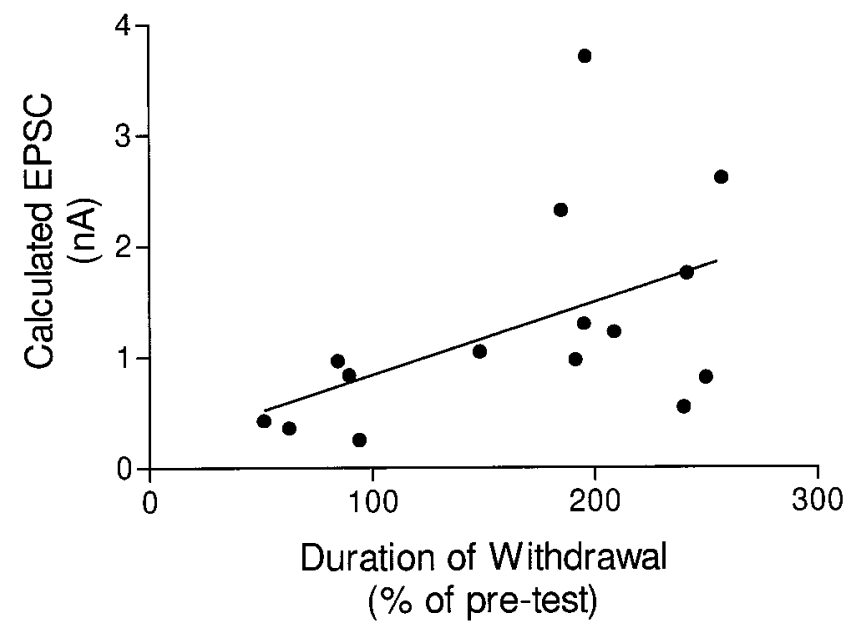

Figure 7. The amplitude of the EPSC from ganglia from the sensitized side was correlated with the duration of withdrawal after sensitization training $(r=0.59)$. Duration was expressed as a percentage of the pretest duration. Values $<100 \%$ indicate that the animal was not sensitized.

physical properties of LP117 were examined: resting membrane potential, input resistance, and excitability (Table 3). None of these properties was affected by long-term sensitization training. The strength of the synaptic connection between LP117 and the follower motor was examined also. A characteristic feature of LP117 is its ability to evoke a slow EPSP in tail motor neurons when it is activated by a series of action potentials (Fig. $8 A, B$ ). The amplitude of the slow EPSP evoked in the motor neuron was not affected by sensitization training (Fig. 8C).

\section{DISCUSSION}

A great deal of information has been obtained regarding defensive withdrawal reflexes in Aplysia. Key emerging concepts are the important contribution of the monosynaptic component of the circuit to the reflex and the important contribution of plasticity at the sensory-motor synapse to modulation of the reflex by learning. These concepts emerged primarily from study of the shortterm forms of learning such as sensitization, habituation, and classical conditioning. It is reasonable to argue that these same concepts also hold for long-term forms of learning. There are, however, very few data available to support that hypothesis.

A key site of plasticity appears to be the afferent limb of the circuit for both short- and long-term forms of sensitization. After delivery of sensitizing stimulation to the skin of a semi-intact preparation or stimulation of the connectives that would convey this information, several short-term changes in biophysical properties of the sensory neurons, expressed as a depolarized membrane potential, increased excitability, and increased action potential duration, have been observed (Walters et al., 1983; Byrne et al., 1990). In addition, there is an increase in the amplitude of the postsynaptic potential evoked in the follower motor neuron by the sensory neuron (Walters et al., 1983; Castellucci et al., 1989).

For long-term sensitization the link between behavioral sensitization and enhancement of the sensorimotor EPSP is less well established. There have been only two studies in which animals were trained and then $24 \mathrm{hr}$ later ganglia were removed from the animal for electrophysiological analysis (Frost et al., 1985; Walters, 1987). In both of these studies the sensorimotor EPSP was enhanced $24 \mathrm{hr}$ after training. Several in vitro analogs of long-term sensitization have been examined in isolated ganglia (Castellucci et al., 1989; Emptage and Carew, 1993; Z hang et al., 1994, 1997) and cell culture (Montarolo et al., 1986; Dale et al., 1987, 1988), demonstrating changes in synaptic strength and excitability of sensory neurons. Changes in sensory neuron morphology also appear to contribute to long-term sensitization (Bailey et al., 1996). In the population of animals studied here, no effects of sensitization training were observed on sensory neuron morphology (Wainwright et al., 1997). Ultrastructural changes were not ruled out, however.

Correlates of long-term habituation have been examined in Aplysia. Synaptic input to motor neurons was reduced (Carew and Kandel, 1973), as was the number of detectable synapses 


\begin{tabular}{|c|c|c|c|}
\hline Correlate & Control & Sensitized & \\
\hline Resting membrane potential & $\begin{array}{l}-58 \mathrm{mV}(9 \mathrm{IR}) \\
(n=11)\end{array}$ & $\begin{array}{l}-60.5 \mathrm{mV}(5 \mathrm{IR}) \\
(n=12)\end{array}$ & $U_{11,12}=82$ \\
\hline Input resistance & $\begin{array}{l}10.7 \mathrm{M} \Omega(5 \mathrm{IR}) \\
(n=11)\end{array}$ & $\begin{array}{l}9.3 \mathrm{M} \Omega(2.6 \mathrm{IR}) \\
(n=12)\end{array}$ & $U_{11,12}=84$ \\
\hline Excitability & $\begin{array}{l}6.5 \text { spikes/nA (1.2 IR) } \\
(n=10)\end{array}$ & $\begin{array}{l}5.9 \text { spikes/nA (0.9 IR) } \\
(n=12)\end{array}$ & $U_{10,12}=80$ \\
\hline
\end{tabular}

A
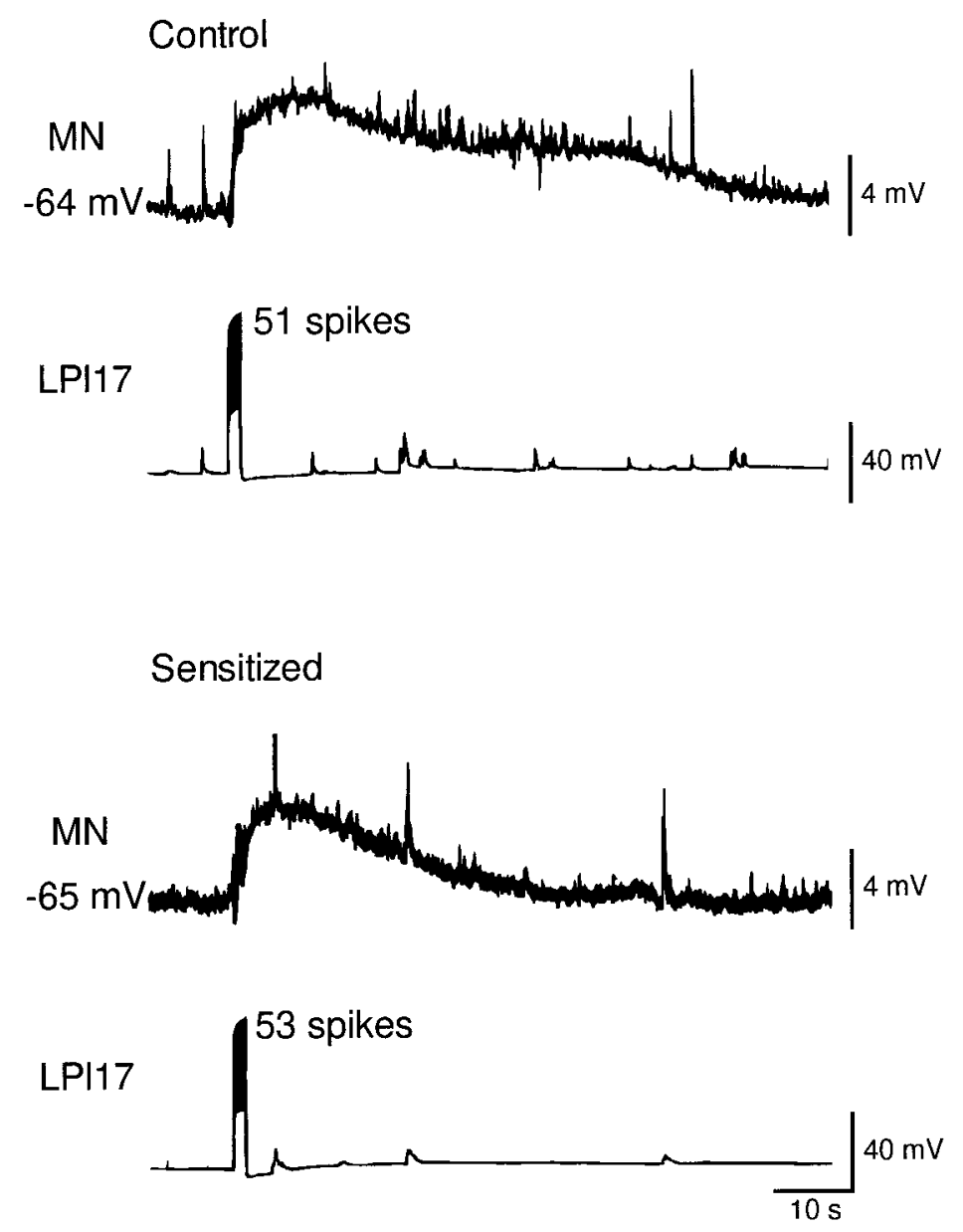

B

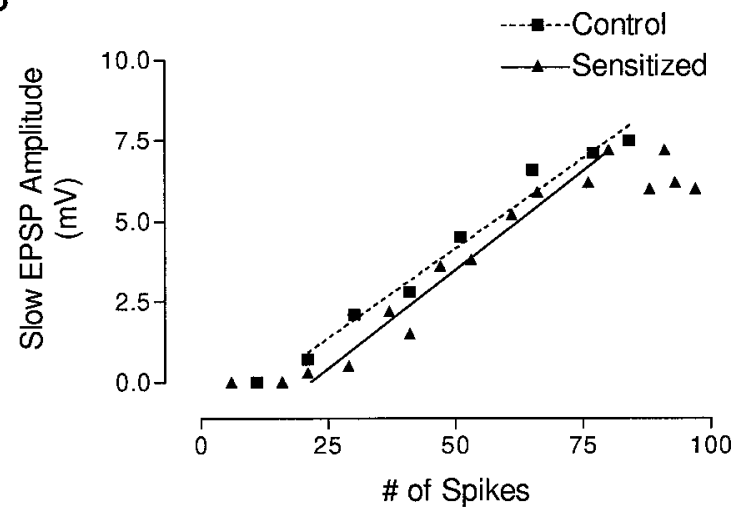

C

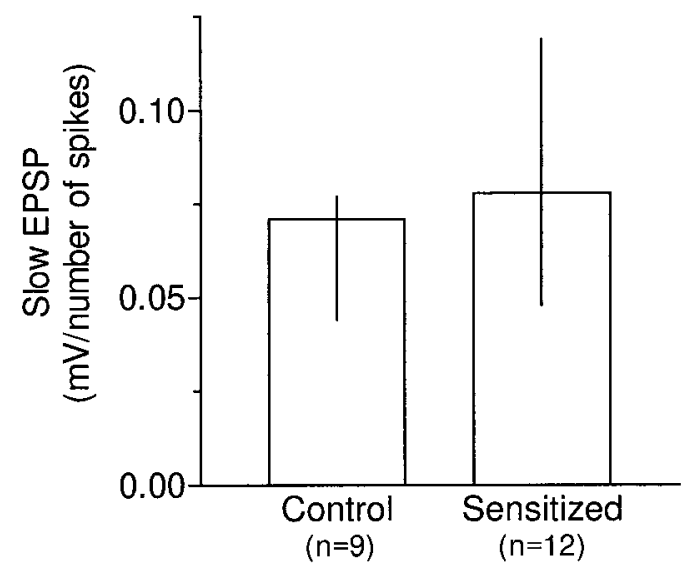

Figure 8. Training had no effect on the amplitude of the slow EPSP evoked in the motor neuron by LP117. Recording of slow EPSPs evoked in ganglia from control (top) and sensitized (bottom) sides of the animal are illustrated in $A$. Because it was difficult to control the number of spikes generated in LP117 by a given current pulse, several slow EPSPs were evoked by depolarizations of different amplitudes. The amplitude of the slow EPSP was plotted against the number of spikes elicited in LP117. The slope of the regression line ( $\mathrm{mV} / \mathrm{spike})$ over the linear range was used to compare ganglia from control and sensitized sides. The relationships for the two preparations shown in $A$ are illustrated in $B$. The slopes of the regression lines are $0.112 \mathrm{mV} / \mathrm{spike}$ (Control) and $0.123 \mathrm{mV} / \mathrm{spike}$ (Sensitized). Group data are illustrated in $C$.

(Castellucci et al., 1978). In the tail-induced siphon withdrawal circuit we did not test for changes in the number of detectable synapses (Frost et al., 1985) because the pleural sensory cluster is, unlike the abdominal LE cluster, structurally heterogeneous, and convergence onto identified motor neurons is low.

In this paper we report a more extensive analysis of the correlates of long-term sensitization of the tail-siphon withdrawal reflex. Several different biophysical properties of sensory neurons, motor neurons, and LP117, an identified interneuron, were exam- ined in animals that had been subjected to long-term sensitization training.

\section{Effects of long-term sensitization training on tail sensory neurons}

Previous work using long-term sensitization training protocols, and supported by in vitro analogs, suggested that tail sensory neurons were an important site of plasticity. Tail sensory neurons demonstrated a persistent decrease of net outward currents after 


\begin{tabular}{|c|c|c|}
\hline Correlate & Correlation coefficient $(r)$ & \\
\hline Synaptic strength of SN-MN synapse & 0.59 & $F_{(1,13)}=7.02^{*}$ \\
\hline Afterdepolarization (50 msec) of SN spike & 0.32 & $F_{(1,15)}=1.69$ \\
\hline Resting membrane potential of $\mathrm{MN}$ & 0.25 & $F_{(1,16)}=1.04$ \\
\hline Threshold membrane potential of MN & 0.19 & $F_{(1,12)}=0.44$ \\
\hline Afterdepolarization $(1 \mathrm{sec})$ of $\mathrm{SN}$ burst & 0.02 & $F_{(1,17)}=0.007$ \\
\hline Excitability $(1 \mathrm{sec})$ of SN & 0.009 & $F_{(1,16)}=0.001$ \\
\hline
\end{tabular}

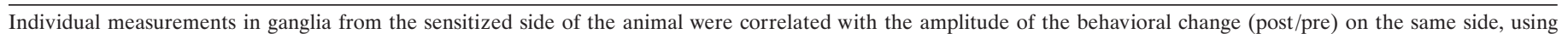
standard linear regression.

*The slope of the regression line was significantly different from $0(p<0.02)$.

behavioral training (Scholz and Byrne, 1987). Moreover, the amplitude of the sensorimotor EPSP was enhanced (Walters, 1987). In the present study both of these effects were confirmed.

Two biophysical properties of tail sensory neurons, membrane excitability and amplitude of the afterdepolarization, were affected by training. Modification of both of these properties is consistent with previous work demonstrating a persistent (24 hr) decrease by training in net outward currents that had kinetics and voltage-sensitivity similar to the S-potassium current (Scholz and Byrne, 1987). The S-current has a strong effect on membrane excitability (Baxter and Byrne, 1989; Byrne et al., 1990) because of its relatively slow kinetics. We do not know the ionic mechanisms of the afterdepolarization and its modulation. One possibility is that the reduction or elimination of the S-current would allow a late inward current to express itself as a slow depolarization.

One consequence of potassium current modulation could be enhanced action potential duration. We did not quantitate action potential duration, because it is difficult to measure this feature accurately when the spike is elicited by the recording electrode. Nevertheless, there were no obvious qualitative changes in action potential duration.

Our results were consistent with the enhancement of the EPSP reported by others for the tail sensory-motor synapse (Walters, 1987) and the siphon sensory-motor synapse (Frost et al., 1985), but the effect was not statistically significant. One possible difference could be the sampling procedures used. In one study only animals that reached a performance criterion were tested (Frost et al., 1985). Moreover, in that study the animals were trained for a longer period of time $(4 \mathrm{~d})$ than in the protocol used here. In another study only the strongest synaptic connections were included in the analysis (Walters, 1987). Both of these procedures could increase the probability of encountering an enhanced synapse in trained animals. We did not use any selection criteria in our analyses, however, and the resultant variability was too large for the difference to achieve statistical significance. When variability was reduced by calculating the EPSC, we observed a significant enhancement. There could be differences in the enhancement induced in specific sensory neurons attributable to heterogeneous modulatory input. For example, some sensory neurons receive denser input from serotonergic pathways than others (Zhang et al., 1991).

One observation that was not confirmed was the occurrence of regenerative bursting in tail sensory neurons (Walters, 1987). In our sample only two cells in 18 ganglia fired multiple action potentials in response to a brief $(50 \mathrm{msec})$ intracellular depolarizing stimulus. One possible explanation is that our training protocol minimized the risk of injury to tail sensory neurons, which causes regenerative bursting (Clatworthy and Walters, 1994). Alternatively, minor differences in the details of the training protocol could have enhanced regenerative spiking selectively.

Although training produced a number of changes, it was interesting to take the analysis one step further and correlate the magnitude of a neuronal property with the magnitude of the behavior. With a linear regression analysis the amplitude of the calculated EPSC was correlated significantly with the duration of the behavior. This is the first study to our knowledge in which this correlation was examined for long-term sensitization in Aplysia. The correlation itself was relatively low $(r=0.59)$, which perhaps is expected, considering the number of synapses in the circuit mediating the tail-elicited siphon response. Surprisingly, the correlation held in trained animals, but not in control animals. This suggests that, in control animals, there are a number of factors regulating siphon withdrawal, but after sensitization the amplitude of the sensory-motor EPSP plays a more prominent role. The causal relationship between EPSP amplitude and duration of contraction is not fully understood. Sensory neuron input appears to contribute only to the initial part of motor neuron activation (Walters et al., 1983; Lieb and Frost, 1997), whereas response duration appears to depend on the activation of interneurons (Cleary and Byrne, 1993; White et al., 1993; Frost and Kandel, 1995; Lieb and Frost, 1997). Therefore, the enhanced EPSP in the motor neuron may reflect enhanced input to interneurons, prolonging the duration of the response. At the behavioral level the amplitude of the gill withdrawal reflex was correlated with the duration of the siphon withdrawal response (Stopfer and Carew, 1987).

Other biophysical changes were not correlated with the duration of siphon withdrawal (Table 4). The lack of correlation does not necessarily rule out a causal role in long-term sensitization, however. For example, lack of correlation with excitability could be attributable to the fact that the $20 \mathrm{msec}$ behavioral test stimulus was too brief to activate the modified processes underlying the enhanced excitability.

\section{Effects of long-term sensitization on tail motor neurons}

Previous studies of long-term sensitization found no effect of training on the biophysical properties of the motor neuron. For example, in siphon motor neurons both the resting membrane potential and the input resistance were unaffected (Frost et al., 1985). In the present study the input resistance of the motor neuron tended to decrease, but the effect was not statistically significant. However, the resting membrane potential was hyperpolarized in motor neurons from the trained side. This effect 
would work against the increased motor neuron activity that presumably underlies the enhanced withdrawal. The hyperpolarization may be compensated for, in part, by a decrease in spike threshold. Thus, modulation of these two biophysical properties of motor neurons may cancel each other effectively. Nevertheless, these observations are important because they demonstrate that the motor neuron is also a site of plasticity. Although minor in themselves, they could indicate that other, more significant, properties of the motor neuron are affected. For example, there are some data to suggest that the morphology of motor neurons is modified by long-term sensitization (Bailey and Chen, 1988). Because the motor neuron fires more readily after sensitization, we would expect to see an increase in excitability. There was a tendency for increased excitability in motor neurons from the sensitized side of the animal, but the increase was not statistically significant.

\section{The role of interneurons in long-term sensitization}

Clearly, the monosynaptic component of the circuit plays a crucial role in mediation and modulation of withdrawal reflexes. Nevertheless, there is strong evidence in Aplysia for a distributed representation of the memory for short-term sensitization (Frost et al., 1988; Trudeau and Castellucci, 1992; Fischer and Carew, 1995; Frost and Kandel, 1995; Wright and Carew, 1995; Xu et al., 1995). Virtually nothing is known of the role of interneurons in long-term sensitization. In the siphon withdrawal circuit a higher percentage of sensory neurons recruited interneurons after longterm sensitization, a finding consistent with the increased strength of the sensorimotor synapse (Frost et al., 1985). In the present study several biophysical properties of the excitatory interneurons LP117 were examined directly. No effects of behavioral training were observed. Moreover, the strength of the slow EPSP evoked in motor neurons by LP117 was not affected by long-term sensitization training.

Although these results suggest that LPl17 is not itself a site of plasticity, enhancement of sensory neuron output to LP117 still could be an important mechanism for increasing motor neuron activation (White et al., 1993). Furthermore, other identified interneurons could be affected by long-term training. For example, modulation of the inhibitory interneurons RPl4 and RPl5 (Buonomano et al., 1992; Xu et al., 1994) may contribute to the behavioral modification. Indeed, mathematical simulations have suggested that inhibition of RP14 would be an effective mechanism for increasing motor neuron activation (Medina et al., 1994). These interneurons are affected by acute application of 5-HT (Xu et al., 1995), raising the intriguing possibility that they also may be a site for long-term sensitization.

In summary, these data confirm and extend the results of others, demonstrating that sensory neurons are a site of plasticity in long-term sensitization. In addition, this is the first study to demonstrate that a second locus is affected by training, the motor neurons. Although the biophysical modifications are modest, they could represent more substantive cellular changes, relating to neurite outgrowth, for example. The interneuron LP117 was not affected by training, but this does not rule out the possibility that other interneurons, such as RP14, are affected. Nevertheless, this work is consistent with the idea that long-term sensitization, like the short-term form, affects multiple sites of plasticity distributed throughout the nervous system.

\section{REFERENCES}

Bailey CH, Chen M (1988) Long-term sensitization in Aplysia increases the number of presynaptic contacts onto the identified gill motor neuron L7. Proc Natl Acad Sci USA 85:9356-9359.

Bailey CH, Bartsch D, Kandel ER (1996) Toward a molecular definition of long-term memory storage. Proc Natl Acad Sci USA 93:13445-13452.

Baxter DA, Byrne JH (1989) Serotonergic modulation of two potassium currents in the pleural sensory neurons of Aplysia. J Neurophysiol 62:665-679.

Buonomano DV, Cleary LJ, Byrne JH (1992) Inhibitory neuron produces heterosynaptic inhibition of the sensory-to-motor neuron synapse in Aplysia. Brain Res 577:147-150.

Byrne JH, Shapiro E, Dieringer N, Koester J (1979) Biophysical mechanisms contributing to inking behavior in Aplysia. J Neurophysiol 42:1233-1250.

Byrne JH, Cleary LJ, Baxter DA (1990) Aspects of the neural and molecular mechanisms of short-term sensitization in Aplysia: modulatory effects of serotonin and cAMP on duration of action potentials, excitability, and membrane currents in tail sensory neurons. In: Symposia medica Hoechst 23: the biology of memory (Squire L, Lindenlaub E, eds), pp 7-28. Stuttgart: Schattauer Verlag.

Carew TJ, Kandel ER (1973) Acquisition and retention of long-term habituation in Aplysia: correlation of behavioral and cellular processes. Science 182:1158-1160.

Castellucci VF, Carew TJ, Kandel ER (1978) Cellular analysis of longterm habituation of the gill withdrawal reflex of Aplysia californica. Science 202:1306-1308.

Castellucci VF, Blumenfeld H, Goelet P, Kandel E (1989) Inhibitor of protein synthesis blocks long-term behavioral sensitization in the isolated gill withdrawal reflex of Aplysia. J Neurobiol 20:1-9.

Clatworthy A, Walters ET (1994) Comparative analysis of hyperexcitability and synaptic facilitation induced by nerve injury in two populations of mechanosensory neurons of Aplysia californica. J Exp Biol 190:217-238.

Cleary LJ, Byrne JH (1993) Identification and characterization of a multifunction interneuron contributing to defensive arousal in Aplysia. J Neurophysiol 70:1767-1776.

Cleary LJ, Byrne JH, Frost WN (1995) Role of interneurons in defensive withdrawal reflexes in Aplysia. Learning Memory 2:133-151.

Dale N, Kandel ER, Schacher S (1987) Serotonin produces long-term changes in the excitability of Aplysia sensory neurons in culture that depend on new protein synthesis. J Neurosci 7:2232-2238.

Dale N, Schacher S, Kandel ER (1988) Long-term facilitation in Aplysia involves increase in transmitter release. Science 239:282-285.

Davis HP, Squire LR (1984) Protein synthesis and memory: a review. Psychol Bull 96:518-559.

Dulin MF, Steffensen I, Morris CE, Walters ET (1995) Recovery of function, peripheral sensitization, and sensory neurone activation by novel pathways following axonal injury in Aplysia californica. J Exp Biol 198:2055-2066.

Emptage NJ, Carew TJ (1993) Long-term synaptic facilitation in the absence of short-term facilitation in Aplysia neurons. Science 262:253-256.

Fischer TM, Carew TJ (1995) Cutaneous activation of the inhibitory L30 interneurons provides a mechanism for regulating adaptive gain control in the siphon withdrawal reflex of Aplysia. J Neurosci 15:762-773.

Frost WN, Kandel ER (1995) Structure of the network mediating siphon-elicited siphon withdrawal in Aplysia. J Neurophysiol 73:2413-2427.

Frost WN, Castellucci VF, Hawkins RD, Kandel ER (1985) Monosynaptic connections made by the sensory neurons of the gill- and siphonwithdrawal reflex in Aplysia participate in the storage of long-term memory for sensitization. Proc Natl Acad Sci USA 82:8266-8269.

Frost WN, Clark GA, Kandel ER (1988) Parallel processing of shortterm memory for sensitization in Aplysia. J Neurobiol 19:297-334.

Goldsmith JR, Byrne JH (1993) Bag cell extract inhibits tail-siphon withdrawal reflex, suppresses long-term but not short-term sensitization, and attenuates sensory-to-motor neuron synapses in Aplysia. J Neurosci 13:1688-1700.

Klein M, Hochner B, Kandel ER (1986) Facilitatory transmitters and cAMP can modulate accommodation as well as transmitter release in Aplysia sensory neurons: evidence for parallel processing in a single cell. Proc Natl Acad Sci USA 83:7994-7998.

Lieb Jr JR, Frost WN (1997) Realistic simulation of the Aplysia siphon- 
withdrawal reflex circuit: roles of circuit elements in producing motor output. J Neurophysiol 77:1249-1268.

Medina JF, Byrne JH, Baxter DA (1994) Role of inhibitory interneurons in a computational model of the tail withdrawal circuit in Aplysia. Soc Neurosci Abstr 20:815.

Mirolli M, Gorman ALF (1968) Abolition of nerve sheath contraction by glutaraldehyde. Comp Biochem Physiol 251:743-746.

Montarolo PG, Goelet P, Castellucci VF, Morgan J, Kandel ER, Schacher S (1986) A critical period for macromolecular synthesis in long-term heterosynaptic facilitation in Aplysia. Science 234:1249-1254.

Pinsker HM, Hening WA, Carew TJ, Kandel ER (1973) Long-term sensitization of a defensive withdrawal reflex in Aplysia. Science 182:1039-1042.

Scholz KP, Byrne JH (1987) Long-term sensitization in Aplysia: biophysical correlates in tail sensory neurons. Science 235:685-686.

Stopfer M, Carew TJ (1987) Quantitative analysis of the relation between gill amplitude and siphon duration in the defensive withdrawal reflex of Aplysia. Behav Neurosci 101:292-295.

Trudeau L-E, Castellucci VF (1992) Contribution of polysynaptic pathways in the mediation and plasticity of Aplysia gill and siphon withdrawal reflex: evidence for differential modulation. J Neurosci 12:3838-3848.

Wainwright M, Zhang H, Byrne JH, Cleary LJ (1997) Morphological correlates of long-term sensitization training in pleural sensory neurons of Aplysia. Soc Neurosci Abstr 23:1334.

Walters ET (1987) Multiple sensory neuron correlates of site-specific sensitization in Aplysia. J Neurosci 7:408-417.

Walters ET, Byrne JH, Carew TJ, Kandel ER (1983) Mechanoafferent neuron innervating tail of Aplysia. I. Response properties and synaptic connections. J Neurophysiol 50:1522-1542.

White JA, Ziv I, Cleary LJ, Baxter DA, Byrne JH (1993) The role of interneurons in controlling the tail-withdrawal reflex in Aplysia: a network model. J Neurophysiol 70:1777-1786.

Wright WG, Carew TJ (1995) A single identified interneuron gates tail shock-induced inhibition in the siphon withdrawal reflex of Aplysia. J Neurosci 15:790-797.

Xu Y, Cleary LJ, Byrne JH (1994) Identification and characterization of pleural neurons that inhibit tail sensory neurons and motor neurons in Aplysia: correlation with FMRFamide immunoreactivity. J Neurosci 14:3565-3577.

Xu Y, Pieroni J, Cleary LJ, Byrne JH (1995) Modulation of an inhibitory interneuron in the neural circuitry for the tail withdrawal reflex of Aplysia. J Neurophysiol 73:1313-1318.

Zhang F, Goldsmith JR, Byrne JH (1994) Neural analogue of long-term sensitization training produces long-term ( 24 and $48 \mathrm{hr}$ ) facilitation of the sensory-to-motor neuron connection in Aplysia. J Neurophysiol 72:778-784.

Zhang F, Endo S, Cleary LJ, Eskin A, Byrne JH (1997) Role of transforming growth factor- $\beta$ in long-term synaptic facilitation in Aplysia. Science 275:1318-1320.

Zhang H, Byrne JH, Cleary LJ (1993) Topographical organization of sensory neurons in the ventrocaudal cluster of the pleural ganglion of Aplysia. Soc Neurosci Abstr 19:813.

Zhang ZS, Fang B, Marshak DW, Byrne JH, Cleary LJ (1991) Serotonergic varicosities make synaptic contacts with pleural sensory neurons of Aplysia. J Comp Neurol 311:259-270. 